\title{
Allergenic activity of Pseudoterranova decipiens (Nematoda: Anisakidae) in BALB/C mice
}

\author{
Alessandra Ludovisi', Gabriella Di Felice', Noelia Carballeda-Sangiao², Bianca Barletta', Cinzia Butteroni', \\ Silvia Corinti', Gianluca Marucci', Miguel González-Muñoz², Edoardo Pozio and Maria Angeles Gómez-Morales ${ }^{1 *}$
}

\begin{abstract}
Background: Anisakis simplex is the only fishery-product associated parasite causing clinical allergic responses in humans so far. However, other anisakids, due to the presence of shared or own allergens, could also lead to allergic reactions after sensitization. The aim of this study was to determine if Pseudoterranova decipiens belonging to the family Anisakidae has allergenic activity and is able to induce sensitization after oral administration in a murine (BALB/c mice) model.

Results: The ingestion of $A$. pegreffii proteins by BALB/C mice, which had been previously sensitized by intraperitoneal inoculation with the corresponding live L3 larvae, triggers signs of allergy within $60 \mathrm{~min}$, whereas $P$. decipiens did to a lesser extent. Beside symptoms, allergic reactions were furtherly supported by the presence of histamine in sera of sensitized mice. Specific $\lg G 1$ and $\lg E$ responses were detected in sera of all sensitized mice from week four. Specific IgG2a response was detected in sera from mice sensitized to $P$. decipiens. After polyclonal or specific activation with anti-CD3/anti-CD28 or antigens, respectively, splenocytes from mice infected i.p. with A. pegreffii or P. decipiens larvae showed significantly higher production of IL-10 than naïve mice. After stimulation with specific antigens, significantly higher IL-5 and IL-13 amounts were produced by specific antigen stimulated splenocytes than by the naive cells; only P. decipiens proteins induced IFN- $\gamma$.
\end{abstract}

Conclusions: The overall results suggest that infection with $P$. decipiens can sensitize mice to react to subsequent oral challenge with anisakid proteins, as described for A. simplex (sensu stricto) and A. pegreffii infections. The results show that anisakid proteins induce a dominant Th2 response, although $P$. decipiens could also induce a mixed type 1/type 2 pattern.

Keywords: Anisakidae, Pseudoterranova decipiens, Anisakis pegreffii, Sensitization, Allergic reactions, BALB/c mice, Immune response

\section{Background}

The family Anisakidae includes marine nematodes, which use marine mammals as primary definitive hosts. The anisakid third-stage larvae (L3) infect fish, mollusks and crustaceans, and consequently, humans may become accidental hosts if they consume raw or undercooked fish [1]. The resulting disease, named anisakidosis, can induce from a mild to severe pathology. This infection is

\footnotetext{
* Correspondence: mariaangeles.gomezmorales@iss.it

'Department of Infectious Diseases, Istituto Superiore di Sanità, viale Regina Elena 299, 00161 Rome, Italy

Full list of author information is available at the end of the article
}

often associated with acute gastrointestinal symptoms such as abdominal pain, diarrhea, nausea, vomiting and in some cases, with allergic reactions [2-4]. The family Anisakidae includes at least 24 genera, of which the more studied are Anisakis, Pseudoterranova and Contracaecum [5]; species of these genera appear to have similar life-cycles, and to share some antigens [4] although their host species vary [6-8]. So far, Anisakis simplex (sensu lato), i.e. Anisakis simplex (sensu stricto), Anisakis pegreffii and Anisakis berlandi are the only fishery-product associated parasites causing clinical allergic responses recognized by EFSA [9]. In fact, $A$. 
simplex (s.l.) exposure may lead to clinical signs as urticaria, rhinitis, bronco-constriction, cough and/or gastrointestinal responses. Acute allergic reactions can also be seen, including anaphylactic shock $[2,4,7,10]$. The potential for type I hypersensitivity responses in acute anisakiasis is indicated by the fact that serum anti- $A$. simplex IgE levels increase rapidly during the first few days, moreover lesions of a nature consistent with type I, type III and type IV hypersensitivity reactions have been found in guinea pigs and rabbits orally infected with Anisakis spp. [2]. Murine models of allergy to A. simplex (s.l.), that mimic the human A. simplex (s.l.) allergy, have been developed to study the specific aspects of anaphylaxis induced by this parasite [11-16]. However, the involvement in allergic responses of anisakid species different from A. simplex (s.l.) has been to our knowledge neglected [17]. The aim of the present study was to determine if Pseudoterranova decipiens belonging to the family Anisakidae, has allergenic activity and is able to induce sensitization.

\section{Methods}

\section{Parasite isolation and identification}

Third-stage larvae (L3) of A. simplex (s.l.) were collected from a silver scabbard fish (Lepidopus caudatus) fished in the Mediterranean Sea and L3 of Pseudoterranova sp. were collected from Atlantic cods (Gadus morhua) fished in the North Atlantic Ocean. L3 were extensively washed one by one in $2 \%$ acetic acid-phosphate buffered saline (PBS) and then each larva was cut into two parts, one part was kept in $90 \%$ ethanol for the molecular identification by PCR/RLPF [18], and the other part was frozen at $-20{ }^{\circ} \mathrm{C}$. L3 from the silver scabbard fish were identified as $A$. pegreffii, whereas L3 from the Atlantic cods were identified as $P$. decipiens. Crude worm extracts (CWE) were prepared from frozen L3. Briefly, 50 frozen L3 were suspended in $0.5 \mathrm{ml}$ of PBS and then homogenized 5 times for $30 \mathrm{~s}$ and sonicated in ice 5 times for $60 \mathrm{~s}$. The suspension was left overnight at $4{ }^{\circ} \mathrm{C}$ under magnetic stirring and further centrifuged at $1500 \times g$ and at $4{ }^{\circ} \mathrm{C}$ for $15 \mathrm{~min}$. The protein content of the centrifuged supernatant was measured by the Bradford method (Quick Start ${ }^{\mathrm{Tn}}$ Bradford, BIO-RAD, Hercules, CA, USA). Moreover, 140 and 80 additional live L3 assumed to be $A$. pegreffii and $P$. decipiens, respectively, on the basis of their morphology [19], host and geographical origins, were collected to be injected into $\mathrm{BALB} / \mathrm{c}$ mice.

\section{Experimental BALB/c model Infection and immunization}

Forty-nine eight-week old female BALB/c mice (20 $\pm 2 \mathrm{~g})$ were housed in the Animal Care Unit of the Istituto
Superiore di Sanità (ISS), Rome, Italy. Experiments were carried out according to the European Directive 63/2010.

To determine the allergenic activity of $A$. pegreffii antigens, 20 mice were allocated into 4 groups of 5 mice each (Table 1). Mice from the first group (1Ap) were orally infected with 2 live $A$. pegreffii L3 using an oral dosing curved cannula for gavage (16G) at week 0 , then re-infected with 2 live $A$. pegreffii $\mathrm{L} 3$ at week 8 , and orally challenged with $5 \mathrm{mg} / \mathrm{mouse}(0.25 \mathrm{mg} /$ mouse g) of CWE from A. pegreffii (ApCWE) in a total volume of $200 \mu \mathrm{l}$ of PBS at week 12. Mice from the second group (2Ap) were anesthetized with $50 \mathrm{mg} / \mathrm{kg}$ ketamine (Ketavet $^{\circ}$, Pfizer, Berlin, Germany) and $3 \mathrm{mg} / \mathrm{kg}$ xylazine (Rompun, Bayer Health Care, Germany); then, the abdominal skin was held and raised by a forceps and mice were injected intraperitoneally (i.p.) with 2 live $A$. pegreffii L3 at week 0 , re-injected i.p. at week 8 , and orally challenged with $5 \mathrm{mg} / \mathrm{mouse}$ of ApCWE at week 12 . Mice from the third group (3Ap) were orally immunized with $700 \mu \mathrm{g} /$ mouse of ApCWE at week 0 and week 8, and then orally challenged with $5 \mathrm{mg} /$ mouse of ApCWE at week 12. Mice from the fourth group (4Ap) used as control, were orally inoculated with PBS following the same protocol as the other experimental groups (Table 1). Allergic reactions were evaluated after the oral challenge by the following scoring system: 0 , no symptom; 1 , scratching and rubbing around the nose and head, hypersensitivity to touch, irritability/aggression; 2, diarrhea, puffiness around the eyes and mouth, pilar erecti, reduced activity, and/or decreased activity with increased respiratory rate; 3 , labored respiration, cyanosis around mouth and tail; 4, loss of consciousness; and 5, death [14].

To determine the allergenic activity of $P$. decipiens antigens, 15 mice were allocated into 3 groups of 5 mice each (Table 1). Mice of the first group (1Pd) were anesthetized as above reported; then mice were injected i.p. with 2 live $P$. decipiens $\mathrm{L} 3$ at week 0 , re-injected at week 8 , and orally challenged with $5 \mathrm{mg} /$ mouse of PdCWE at week 12 . Mice of the second group (2Pd) were orally immunized on day 0 with $700 \mu \mathrm{g} / \mathrm{mouse}$ of PdCWE, and then re-inoculated with the same dose at week 8 . At week 12 , mice were orally challenged with $5 \mathrm{mg}$ /mouse of PdCWE. Mice from the third group (3Pd) used as control, were orally inoculated with PBS following the same protocol that the other experimental groups (Table 1). Allergic reactions were evaluated after the oral challenge as above reported for $A$. pegreffii.

\section{Sera drawing and specific antibodies and histamine in serum}

Blood samples were taken from each mouse by lateral tail vein bleeding at $0,4,8,12$ weeks and at 60 min after oral challenge. Individual sera were stored at $-20{ }^{\circ} \mathrm{C}$ until analysis. Specific antibody responses (IgG1, IgG2a and 
Table 1 Experimental model of allergic sensitization and anaphylactic response in BALB/c mice with Anisakis pegreffii or Pseudoterranova decipiens

\begin{tabular}{|c|c|c|c|c|c|}
\hline $\begin{array}{l}\text { Mice group } \\
\text { ( } 5 \text { mice per group) }\end{array}$ & Infection & Re-infection & Challenge & $\begin{array}{l}\text { Evaluation of allergic } \\
\text { reactions } 60 \text { min after } \\
\text { the last challenge }\end{array}$ & $\begin{array}{l}\text { Evaluation of the immune } \\
\text { responses } 24 \mathrm{~h} \text { after the } \\
\text { last challenge }\end{array}$ \\
\hline \multicolumn{6}{|l|}{ Anisakis pegreffii } \\
\hline $1 \mathrm{Ap}$ & 2 L3 per os & $2 \mathrm{~L} 3$ per os & 5 mg CWE per os & yes & yes \\
\hline \multirow[t]{2}{*}{$2 \mathrm{Ap}$} & 2 L3 i.p. & 2 L3 i.p. & $5 \mathrm{mg}$ CWE per os & yes & yes \\
\hline & Inoculation & Challenge & Challenge & & \\
\hline $3 \mathrm{Ap}$ & $700 \mu \mathrm{g}$ CWE per os & $700 \mu \mathrm{g}$ CWE per os & 5 mg CWE per os & yes & yes \\
\hline $4 \mathrm{Ap}$ & PBS per os & PBS per os & PBS per os & yes & yes \\
\hline Week p.i. & 0 & 8 & 12 & 12 & 12 \\
\hline \multicolumn{6}{|c|}{ Pseudoterranova decipiens } \\
\hline & Infection & Re-infection & Challenge & & \\
\hline \multirow[t]{2}{*}{$1 \mathrm{Pd}$} & 2 L3 i.p. & 2 L3 i.p. & $5 \mathrm{mg}$ CWE per os & yes & yes \\
\hline & Inoculation & Challenge & Challenge & & \\
\hline $2 \mathrm{Pd}$ & $700 \mu \mathrm{g}$ CWE per os & $700 \mu \mathrm{g}$ CWE per os & 5 mg CWE per os & yes & yes \\
\hline $3 \mathrm{Pd}$ & PBS per os & PBS per os & PBS per os & yes & yes \\
\hline Week p.i. & 0 & 8 & 12 & 12 & 12 \\
\hline
\end{tabular}

IgE) were evaluated in individual serum samples by an in-house ELISA. Plates (Greiner Bio-One, Frickenhausen, Germany) were coated with CWE $(5 \mu \mathrm{g} / \mathrm{ml})$ from $A$. pegreffii or $P$. decipiens, and sera were diluted 1:20 (IgG2a and IgE) or 1:200 (IgG1). Results were expressed as Optical Density (OD). Histamine levels in the serum samples taken $60 \mathrm{~min}$ after the challenge [14], were measured by an enzyme immunoassay kit (IBL International, Hamburg, Germany).

Cell cultures and cytokine levels in culture supernatants The day after the evaluation of the allergic reactions, mice were sacrificed, and spleens were harvested under sterile conditions. After spleen disruption by a syringe and erythrocyte lysis, splenocytes were re-suspended in RPMI 1640 (Invitrogen Life Technologies, Gaithersburg, MD) supplemented with 10\% FBS (Hyclone Laboratories, Logan, UT), $1 \mathrm{mM}$ sodium pyruvate, $0.1 \mathrm{mM}$ nonessential amino acids, $2 \mathrm{mM}$ L-glutamine, $25 \mathrm{mM}$ HEPES, $100 \mathrm{U} / \mathrm{ml}$ penicillin, $100 \mathrm{mg} / \mathrm{ml}$ streptomycin (all Invitrogen Life Technologies), and 0.05 mM 2-ME (Merck, Darmstadt, Germany) (complete medium) at a final concentration of $2.5 \times 10^{6}$ cells $/ \mathrm{ml}$ (24-well plates in duplicate, Costar Corporation, Cambridge, MA) for cytokine analysis. Cell cultures were stimulated with $50 \mu \mathrm{g} / \mathrm{ml}$ of CWE for 6 days or with anti-CD3 $(10 \mu \mathrm{g} / \mathrm{ml})$ and soluble anti-CD28 $(2 \mu \mathrm{g} / \mathrm{ml})$ monoclonal antibodies (BD Biosciences Pharmingen, San Josè, $\mathrm{CA}$ ) for 3 days. At the end of the incubation period, supernatants were harvested for the cytokine (Interleukin (IL)-4, IL-5, IL-10 and Interferon (IFN)- $\mathrm{\gamma}$ ) analysis and stored at $-70{ }^{\circ} \mathrm{C}$ until assayed. The cytokine (IL-4, IL-5, IL-10 and IFN-y) production was determined by commercial ELISA kits (eBioscience Affimetrix, San Diego, CA), according to the manufacturer's instructions.

\section{Statistical analysis}

The Mann-Whitney U-test was used to compare each experimental group with the relevant control group. $P$-value $<0.05$ was considered significant.

\section{Results}

Anisakis pegreffii induces allergic symptoms in BALB/C mice, whereas $P$. decipiens does to a lesser extent

The gavage of $A$. pegreffii proteins to previously sensitized mice triggered signs of allergy within $60 \mathrm{~min}$. The maximum symptom score (diarrhoea, reduced activity, and/or decreased activity with increased respiratory rate and cyanosis around the tail) was observed in animals injected i.p. with live L3. The ingestion of $P$. decipiens proteins in previously sensitized mice, induced irritability and reduced activity within $60 \mathrm{~min}$, similarly to $A$. pegreffii (Fig. 1a). Differences in scoring among the $A$. pegreffii experimental groups were observed, whereas no difference was detected between the $P$. decipiens experimental groups. These results were further supported by the histamine presence in sera from all groups of mice inoculated with $A$. pegreffii, in which a higher histamine release than in the naïve control group $(P<0.05)$, was observed (Ap1: $U_{(0)}=2, Z=2.50, P=0.0118$; Ap2: $\left.U_{(0)}=2, Z=3.59, P=0.0001\right)$. In $P$. decipiens sensitized mice, no significantly different histamine release was 


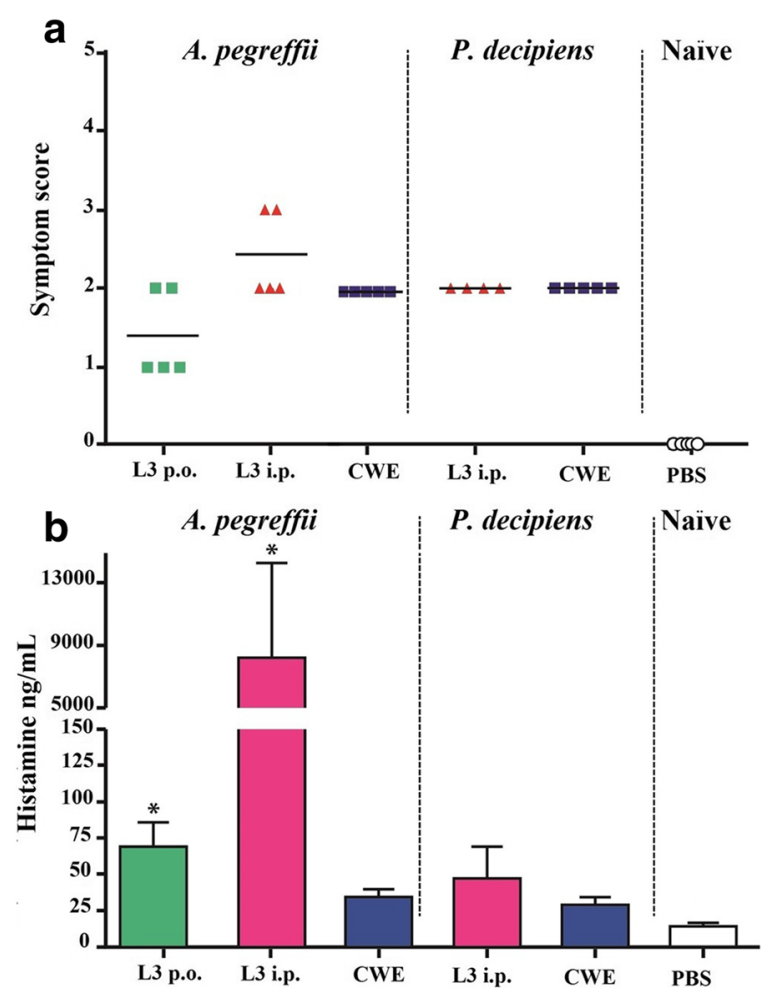

Fig. 1 Allergic reactions in Anisakis pegreffii and Pseudoterranova decipiens sensitized mice. a Symptoms score in BALB/c mice. b Histamine release in BALB/C mouse sera. Green symbols and histograms: mice orally infected with $A$. pegreffii ( $L 3$ p.o.); red symbols and histograms: mice intraperitoneally infected with $A$. pegreffii or P. decipiens (L3 p.i.); blue symbols and histograms: mice orally immunized with $A$. pegreffii or $P$. decipiens crude worm extracts (CWE); open circles and histogram: mice (naïve) intraperitoneally inoculated with phosphate buffered saline (PBS). Symbols represent individual mice (combined results of 2-3 experiments). Horizontal bars represent medians. Reactions were scored following the protocol of Nieuwenhuizen et al. [14]. Histograms represent mean values with SE. Significances $\left({ }^{*} P<0.05\right)$ were calculated between experimental and naive control groups. Each experiment was conducted in triplicate

observed in comparison with the naïve control group (Fig. 1b). A certain variability among individual responses was observed in all groups of mice, despite inbred BALB/C mice were used.

The i.p. infection of BALB/C mice with $A$. pegreffii $\mathrm{L} 3$ induces a dominant Th2 immune response, whereas $P$. decipiens induces a mixed Th1/Th2 response

Polyclonal stimulation of splenocytes from mice inoculated i.p. with $A$. pegreffii L3 (group 2Ap) with antiCD3/anti-CD28 induced significantly $\left(U_{(0)}=2, Z=2.50\right.$, $P=0.01208$ ) higher levels of IL-5 and IL-10 than in splenocytes from mice of $1 \mathrm{Ap}$ and $3 \mathrm{Ap}$ groups and naïve mice (Fig. 2). Splenocytes from mice inoculated i.p. with P. decipiens L3 (group 1Pd) produced IL-10 at a significantly $\left(U_{(0)}=2, Z=2.50, P=0.01208\right)$ higher level than that of naive mice (Fig. 2). After activation with anti-CD3/anti-CD28, splenocytes from all $A$. pegreffii experimental mouse groups produced significantly (1Ap and Ap2: $U_{(0)}=2, Z=2.50, P=0.01208$; Ap3 $=U_{(1)}=2$, $Z=2.29, P=0.02114$ ) lower IFN-y levels than those from the non-exposed naïve mice.

The antigen-specific stimulation of splenocytes from mice injected i.p. with $A$. pegreffii L3 (group 2Ap) induced significantly higher levels of IL-5, IL-10 and IL-13 than those produced by splenocytes from naïve mice (IL-5 and IL-10: $U_{(0)}=2, Z=2.50, P=0.01208$; IL-13: $U_{(0)}=8, Z=3.000, P=0.027$ ) (Fig. 3). The stimulation of splenocytes from mice injected i.p. with $P$. decipiens

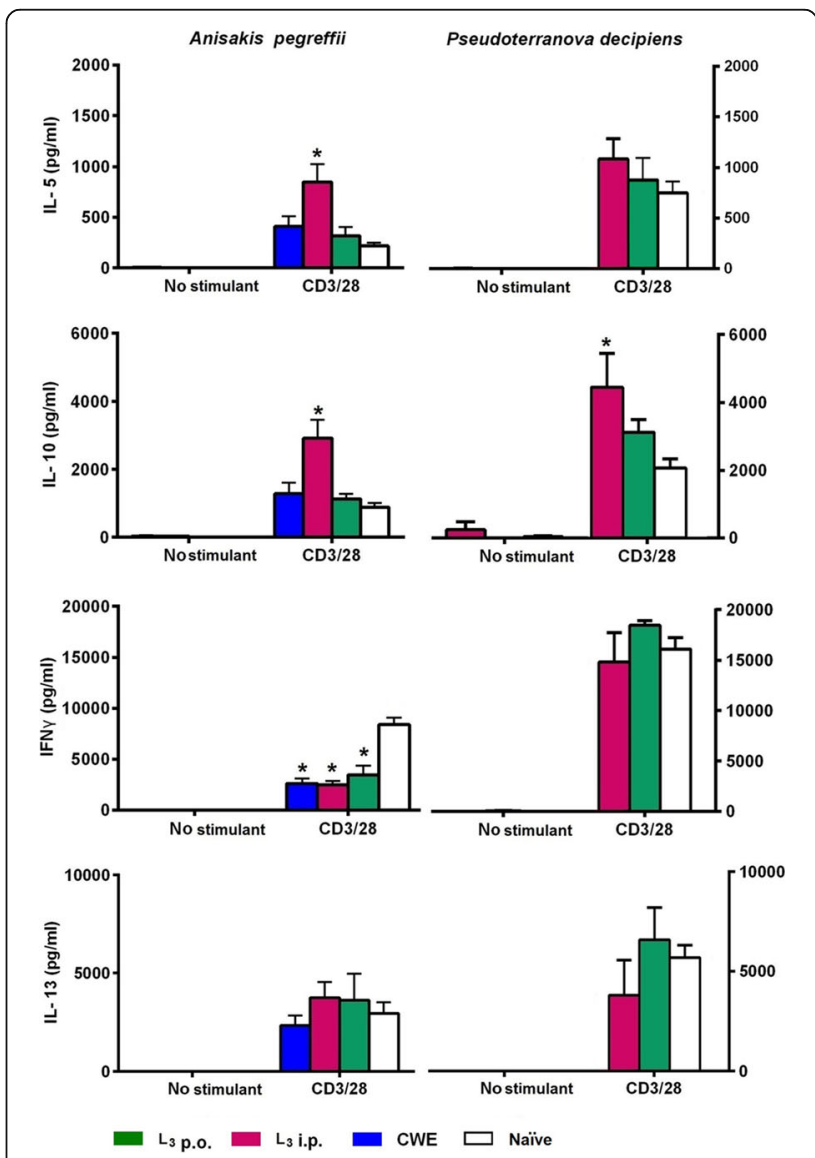

Fig. 2 Cytokine production in supernatants of splenocyte cultures stimulated with anti-CD3 and anti-CD28 monoclonal antibodies at $10 \mu \mathrm{g} / \mathrm{ml}$ and $2 \mu \mathrm{g} / \mathrm{ml}$, respectively. Splenocytes were harvested from Anisakis pegreffii or Pseudoterranova decipiens infected BALB/C mice at week 12 post - infection. Green histogram: mice orally infected with 2 A. pegreffii $\mathrm{L} 3$ ( $\mathrm{L} 3$ p.o.); red histogram: mice intraperitoneally infected with 2 A. pegreffii $L 3$ or 2 P. decipiens $L 3$ ( $L 3$ p.i.); blue histograms: mice orally inoculated with $A$. pegreffii or $P$. decipiens crude worm extracts (CWE); open histogram: mice intraperitoneally inoculated with phosphate buffered saline (PBS). Significances $\left({ }^{*} P<0.05\right)$ were calculated between experimental and PBS control groups. Each experiment was conducted in triplicate 


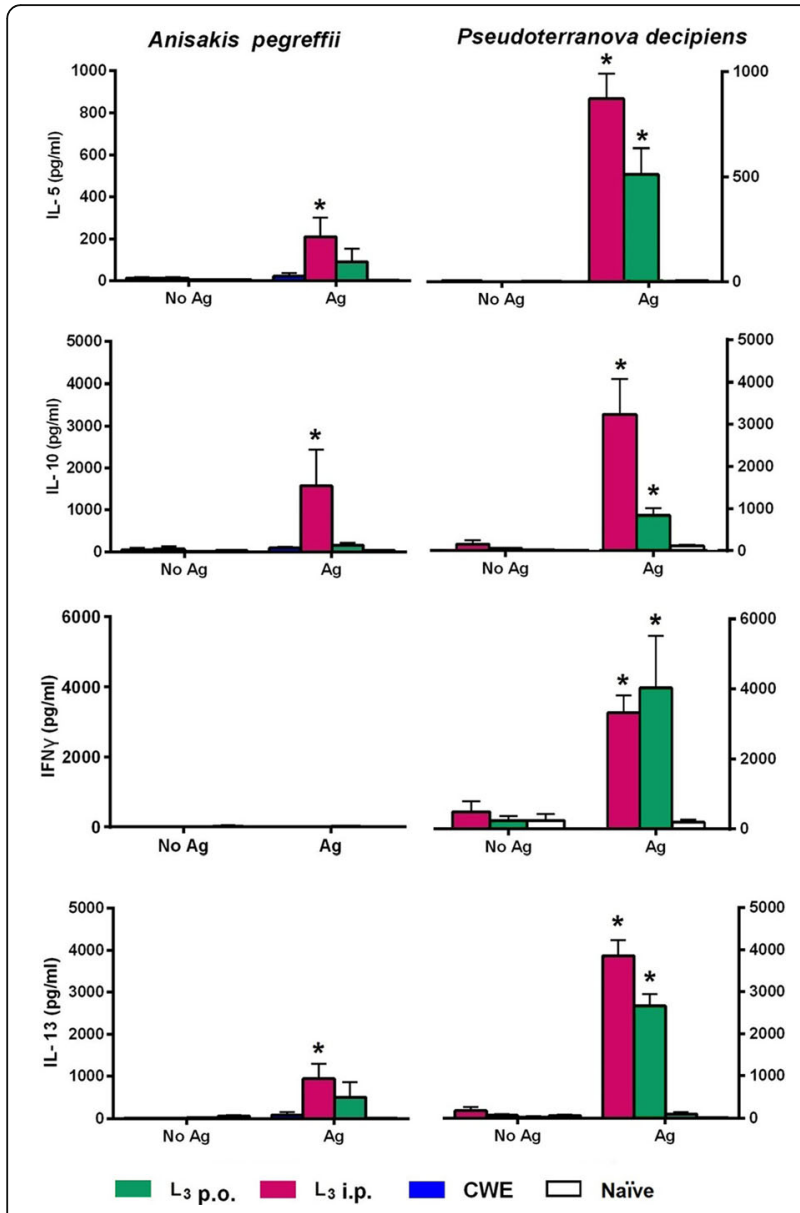

Fig. 3 Cytokine production in supernatants of splenocyte cultures stimulated with Anisakis pegreffii or Pseudoterranova decipiens crude worm antigens (CWE). Splenocytes from BALB/c mice were harvested at week 12. Green histogram: mice orally infected with 2 A. pegreffii L3 (L3 p.o.); red histogram: mice intraperitoneally infected with 2 A. pegreffii $L 3$ or 2 P. decipiens $L 3$ ( $L 3$ p.i.); blue histograms: mice orally inoculated with $A$. pegreffii CWE or $P$. decipiens CWE (CWE); open histogram: mice intraperitoneally inoculated with phosphate buffered saline (PBS). Abbreviations: No Ag, nonstimulated splenocytes; Ag, splenocytes stimulated with specific CWE. Significances $\left({ }^{*} P<0.05\right)$ were calculated between experimental and PBS control groups. Each experiment was conducted in triplicate

L3 (group 1Pd) and mice orally inoculated with CWE from $P$. decipiens (group $2 \mathrm{Pd}$ ), induced significantly higher levels of IL-5, IL-10, IL-13 and IFN- $\gamma$ than those produced by the splenocytes obtained from naïve mice $\left(U_{(0)}=2, Z=2.50, P=0.01208\right)$ (Fig. 3$)$.

The intraperitoneal exposure of BALB/c mice to both A. pegreffii L3 and $P$. decipiens L3, induced specific IgE and IgG1 levels in mouse sera from week 8 and 4, respectively, until the last day of observation. Detectable IgG2a levels were found only in the sera from mice i.p. exposed to $P$. decipiens L3 (Fig. 4).

\section{Discussion}

The results suggest that infection with $P$. decipiens is able to sensitize mice to react to subsequent oral challenge with anisakid proteins, as occurs with $A$. pegreffii infection, and that anisakid proteins induce a dominant Th2 response, although $P$. decipiens could also induce a mixed Th1/Th2 pattern.

Several experimental models of Anisakis spp. allergic disease were developed in an attempt to elucidate the immunological mechanisms of anisakiasis. These experimental models showed that the Anisakis spp. infection predisposes to allergic responses once the host is challenged with Anisakis spp. proteins. After challenging, specific IgE and IgG1, and sometimes IgG2a, were detected in serum. Moreover, synthesis of Th2 cytokines was prevalent even if sometimes a mixed Th1/Th2 cell response was found [11, 14, 15, 20-24].

These literature data are comparable with the results of the present study, in which BALB/c mice exhibited allergic symptoms after they were infected i.p. with $A$. pegreffii or $P$. decipiens live L3, reinfected to boost responses, and then orally challenged with $5 \mathrm{mg}$ of proteins. In the developed models, beside symptoms, allergic reactions were further supported by the presence of histamine in the serum samples. However, $P$. decipiens i.p. infection induced mild symptoms and lower histamine release than $A$. pegreffii (Fig. 1).

This immune response could be explained by a lower pathogenic and/or allergenic potential of $P$. decipiens than that of A. simplex (s.l.). Human infections caused by anisakid species as Pseudoterranova spp. and Contracaecum spp. or by species from a different family (e.g. Hysterothylacium spp., Raphidascarididae) are reported to be often mild and transient, causing nausea, cramps, pharyngeal irritation and occasionally allergic symptoms [25, 26]. L3 of these genera can occasionally penetrate the gastric tract to cause acute disease, but on the whole, they are less invasive than L3 of species of the genus Anisakis [27, 28]. Regarding allergenic potential, the orogastric infection of mice with live L3 of Contracaecum sp. did not elicit serum sensitization [17]. However, no information was published on Pseudoterranova spp. experimental infections. So far, 14 different proteins have been identified as A. simplex allergens; six of these are recognized by more than $50 \%$ of Anisakis-allergic patients, and then identified as major allergens [29]. Ani s 7, one of the major A. simplex allergens, is not present in, or is antigenically different from Pseudoterranova spp. allergens [30]. An immunogenic haemoglobin of $37.6 \mathrm{kDa}$ from $P$. decipiens was isolated and sequenced; it was not immunologically characterized [31] but presents a high degree of homology with Ani s 13, which is a main allergen of A. simplex (s.l.) [32]. Moreover, the $A$. pegreffii haemoglobin is an IgG- and IgE-reacting protein in Anisakis-infected mice [33]. 


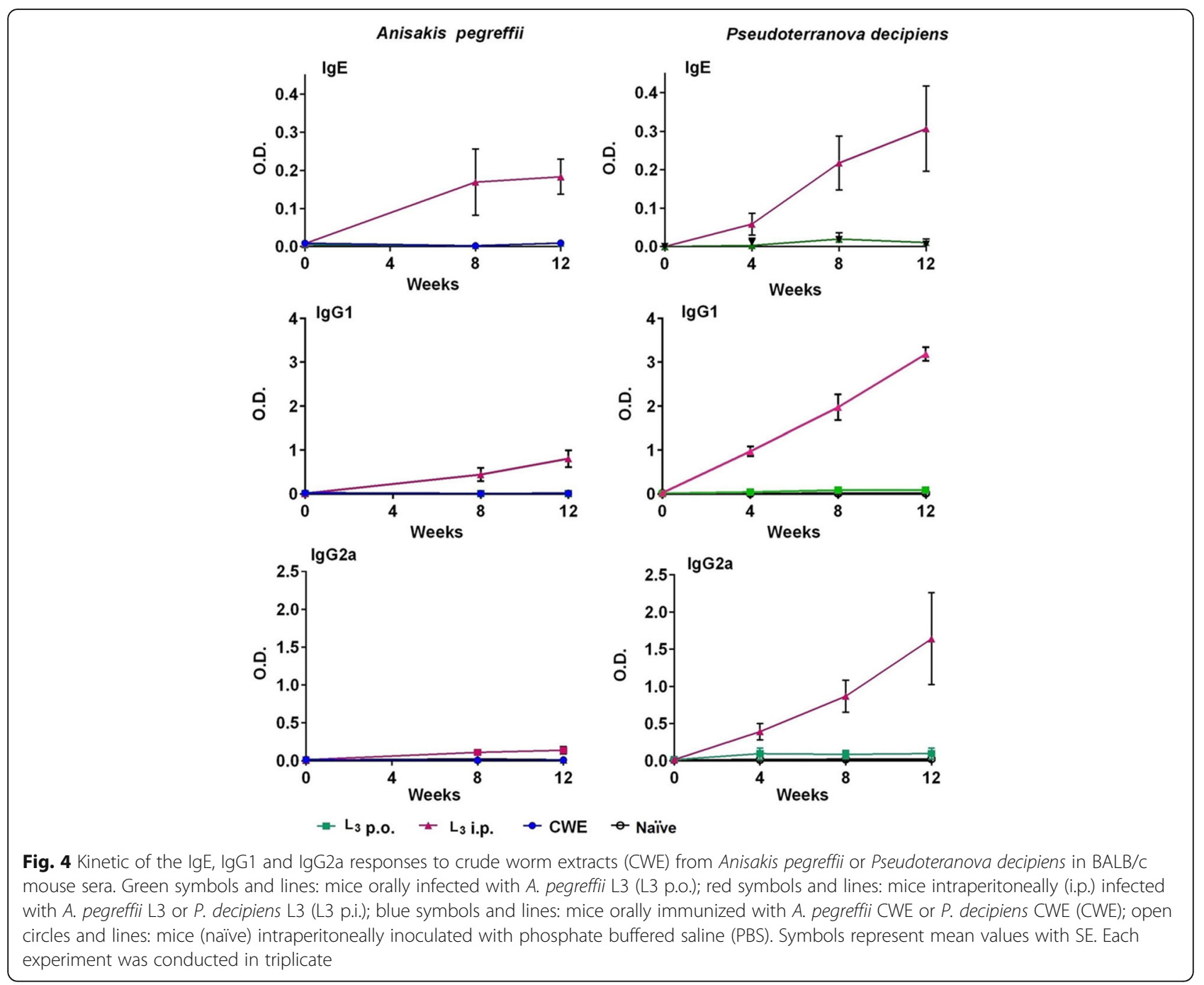

Even if there is no detailed information either on other Pseudoterranova spp. antigens or allergens, we cannot categorically exclude the presence of such molecules in species of this nematode either own or shared with other members of the same family (Anisakidae) or superfamily (Ascaridida), as for A. simplex (s.l.) and Hysterothylacium aduncum [34].

Besides allergenic proteins, the different anisakid genera could be heterogeneous in terms of presence of immunomodulatory molecules. A different pattern of such molecules could be responsible for the final outcome of the immune response, shifting towards a prevalent Th2 or Th1/Th2 polarization. The immunomodulatory properties of these molecules could also account for the different severity of allergic symptoms elicited by the challenge with the two anisakid extracts.

In the present study, differences in scoring and histamine release among the experimental groups of $A$. pegreffii were observed; whereas, no difference was detected between the experimental groups of $P$. decipiens.
For experimental models of anisakid L3 infection, there is a consensus, in which the intraperitoneal infection is the best route of inoculation, since it closely mimics human infection, and it is less traumatic for mice than the oral administration due to the relatively large size of L3. Moreover, by the intraperitoneal route, larvae cannot be discharged, whereas more than $50 \%$ of L3 are discharged in orally-infected mice [35]. In our study, no allergic reaction was observed in mice orally infected with $A$. pegreffii L3. On the basis of literature data, we can argue that the orally introduced L3 could have been expelled.

Several reports suggest that only the ingestion of live larvae, which attach themselves to the gastric mucosa, can predispose to allergic reaction in humans [36-38]. In fact, oral challenges with lyophilized L3 or excretory-secretory extracts from L3 to sensitized patients did not induce allergic manifestations $[38,36]$. The possibility exists that lyophilized L3 lose their allergenic activities. However, Anisakis-associated hypersensitivity cases attributable to 
the ingestion of cooked, canned, and frozen seafood have also been suggested in countries where the consumption of fish infected with Anisakis spp. is highly frequent $[2,39,40]$. Therefore, the allergenic activity of ingested L3 proteins without any treatment, which could alter their allergenic nature, was tested in the present study in mice orally inoculated with $700 \mu \mathrm{g} /$ mouse of ApCWE or PdCWE. The maximum symptom score observed in both mouse groups was not severe and no histamine release was detected after the oral challenge in comparison with the control mice (Fig. 1). Consequently, the oral inoculation followed by the oral challenge with CWEs did not induce allergic sensitization, which was further confirmed by the absence of an IgE response in those mice (Fig. 4). Since the histamine release was detected in mice sensitized with CWE or in orally injected L3 mice, in which specific immunoglobulin responses were not induced, a direct IgE-independent stimulatory effect on mast cells can be argued.

The cytokine pattern produced by splenocytes from mice inoculated i.p. with A pegreffii or P. decipiens L3, which had been activated with anti-CD3/anti-CD28 or with specific antigens, suggests the prominence of a Th2 response (IL-5, IL-10 and IL-13) for the $A$. pegreffii model, and a mixed Th1/Th2 response (IL-5, IL-10, IL13 and IFN-y) for the P. decipiens model (Fig. 2). These results were supported by the mouse humoral immune response (IgE, IgG1 and IgG2a) (Fig. 4). Indeed, P. decipiens induce Th2-associated immune response (IgE and IgG1 antibodies, IL-5, IL-10 and IL-13) and also a Th1like response characterized by high levels of IgG2a antibody and by IFN- $\gamma$ production, whereas in $A$. pegreffii, Th2 and not Th1-associated immune responses are evident. Similar results were obtained with other murine models of infection with $A$. simplex $[11,23]$ or $A$. pegreffii [14], in which the Anisakis sp. infection induced Th2-biased immune responses with elevated synthesis of IgE and IgG1 and an increased splenocyte production of IL-5, IL-10 and IL-13. The systemic Th2 response can predispose to allergic reactions after an oral challenge with Anisakis sp. proteins ending in a type 1 hypersensitivity reaction. Instead, for Pseudoterranova spp., no experimental models have been developed so far. In the $P$. decipiens $\mathrm{BALB} / \mathrm{c}$ animal model described here, a mixed Th1/Th2 pattern was observed, similar to that observed in an $A$. simplex mouse $(\mathrm{C} 3 \mathrm{H} / \mathrm{He})$ model, in which mice were sensitized i.p. and then intravenously challenged with $A$. simplex antigens [13].

In the present study, the same mixed Th1/Th2 cytokine patterns (IL-5, IL-10, IL-13 and IFN- $\mathrm{y}$ ) was produced by splenocytes from mice orally inoculated with $700 \mu \mathrm{g}$ of ApCWE or PdCWE when stimulated with the specific antigens, but no specific antibody response was found in sera from these mice.
A certain intra-group variability among individual responses was observed along the experiments, despite inbred BALB/c mice were used; this variability is not an uncommon finding in allergy models, since it has been also found in a robust model of cockroach allergen induced asthma-like pulmonary inflammation, in which inbred $B A L B / C$ and outbred mice display roughly equivalent levels of overall, intra-strain variability [41].

\section{Conclusions}

The overall results suggest that infection with $P$. decipiens can sensitize mice to react to subsequent oral challenge with anisakid proteins, as described for $A$. simplex (s.s.) and A. pegreffii infections. The results show that anisakid proteins induce a dominant Th2 response, although $P$. decipiens could also induce a mixed type $1 /$ type 2 pattern.

\begin{abstract}
Abbreviations
1Ap: Mice from Anisakis pegreffii group 1; 1Pd: Mice from Pseudoterranova decipiens group 1; 2Ap: Mice from A. pegreffii group 2; 2-ME: 2-

mercaptoethanol; 2Pd: Mice from P. decipiens group 2; 3Ap: Mice from $A$. pegreffii group 3; 3Pd: Mice from $P$. decipiens group 3; 4 Ap: Mice from $A$. pegreffii group 4; Ag: Specific crude worm extract; Ani s: A. simplex allergen; ApCWE: A. pegreffii crude worm extract; CWE: Crude worm extract:

ELISA: Enzyme immunosorbent assay; FBS: Fetal bovine sera;

i.p.: Intraperitoneally; IFN: Interferon; Ig: Immunoglobulin; IL: Interleukin;

ISS: Istituto Superiore di Sanità; L3: Third-stage larvae; OD: Optical density; p.o.: infected per os; PBS: Phosphate buffered saline; PCR/RLPF: PCR/random amplified polymorphic fragments; PdCWE: P. decipiens crude worm extract; SE: Standard error; Th: T-helper
\end{abstract}

\section{Acknowledgments}

We are very grateful to Angel Fernandez González for his willingness to provide Pseudoterranova decipiens live larvae and Marco Amati for his technical assistance. This work was financed by FP7-312068 EU PARASITE.

\section{Availability of data and material}

The datasets used and/or analysed during the current study are available from the corresponding author on reasonable request.

\section{Funding}

This work was financed by FP7-312068 EU PARASITE.

\section{Authors' contributions}

All authors have contributed significantly to this study: AL and NC participated in the study design, collection of samples and interpretation of the results. GD, BB, CB and SC carried out the assays, performed the statistical analysis and participated in the interpretation of the results. GL participated in the experimental model. MG participated in the study design. EP revised critically the manuscript for important intellectual content. MAGM conceived and coordinated the study and draft the manuscript. All authors read and approved the final manuscript.

\section{Competing interests}

The authors declare that they have no competing interests.

\section{Consent for publication}

Not applicable.

\section{Ethics approval}

The Animal Care Committee of the Istituto Superiore di Sanità approved the in vivo protocol $n^{\circ} 8 / 2014-B$ of January 15th 2014 from the Ministry of Health (DL 116/92). 


\section{Publisher's Note}

Springer Nature remains neutral with regard to jurisdictional claims in published maps and institutional affiliations.

\section{Author details}

${ }^{1}$ Department of Infectious Diseases, Istituto Superiore di Sanità, viale Regina Elena 299, 00161 Rome, Italy. ${ }^{2}$ University Hospital La Paz-FIBHULP, Paseo Castellana 261, 28046 Madrid, Spain.

Received: 22 February 2017 Accepted: 4 June 2017

Published online: 12 June 2017

\section{References}

1. Pozio E. Integrating animal health surveillance and food safety: the example of Anisakis. Rev Sci Tech. 2013:32:487-96.

2. Audicana MT, Kennedy MW. Anisakis simplex: from obscure infectious worm to inducer of immune hypersensitivity. Clin Microbiol Rev. 2008;21:360-79.

3. Pravettoni V, Primavesi L, Piantanida M. Anisakis simplex: current knowledge. Eur Ann Allergy Clin Immunol. 2012;44:150-6.

4. Nieuwenhuizen N, Lopata AL. Anisakis - a food-borne parasite that triggers allergic host defences. Int J Parasitol. 2013;43:147-57.

5. Arcos SC, Ciordia S, Roberston L, Zapico I, Jimenez-Ruiz Y, Gonzalez-Munoz $M$, et al. Proteomic profiling and characterization of differential allergens in the nematodes Anisakis simplex sensu stricto and A. pegreffii. Proteomics. 2014;14:1547-68.

6. Mattiucci S, Nascetti G, Clanchi R, Paggi L, Arduino P, Margolis L, et al. Genetic and ecological data on the Anisakis simplex complex, with evidence for a new species (Nematoda, Ascaridoidea, Anisakidae). J Parasitol. 1997;83: 401-16.

7. Audicana MT, Ansotegui IJ, de Corres LF, Kennedy MW. Anisakis simplex: dangerous - dead and alive? Trends Parasitol. 2002;18:20-5.

8. Klapper R, Kuhn T, Münster J, Levsen A, Karl H, Klimpel S. Anisakid nematodes in beaked redfish (Sebastes mentella) from three fishing grounds in the North Atlantic, with special notes on distribution in the fish musculature. Vet Parasitol. 2015:207:72-80

9. EFSA. Scientific opinion on risk assessment of parasites in fishery products. EFSA J. 2010:8:1543.

10. Alonso A, Daschner A, Moreno-Ancillo A. Anaphylaxis with Anisakis simplex in the gastric mucosa. N Engl J Med. 1997;337:350-1.

11. Iglesias R, Leiro J, Ubeira FM, Santamarina MT, Sanmartin ML. Anisakis simplex: antigen recognition and antibody production in experimentally infected mice. Parasite Immunol. 1993;15:243-50.

12. Amano T, Nakazawa M, Sugiyama H, Secor WE, Oshima T. Specific antibody patterns of Wistar rats inoculated with third stage larvae of Anisakis simplex. J Parasitol. 1995;81:536-42.

13. Baeza ML, Conejero L, Higaki Y, Martín E, Pérez C, Infante S, et al. Anisakis simplex allergy: a murine model of anaphylaxis induced by parasitic proteins displays a mixed Th1/Th2 pattern. Clin Exp Immunol. 2005;142:433-40.

14. Nieuwenhuizen N, Lopata AL, Jeebhay MF, Herbert DR, Robins TG, Brombacher F. Exposure to the fish parasite Anisakis causes allergic airway hyperreactivity and dermatitis. J Allergy Clin Immunol. 2006;117:1098-105.

15. Cho SW, Lee HR. Immune reactions and allergy in experimental anisakiasis. Korean J Parasitol. 2006;44:271-83.

16. Abdel-Ghaffar F, Mahmoud Badr A, Morsy K, Ebead S, El Deeb S, Al Quraishy $S$, et al. Cytokine signature and antibody-mediated response against fresh and attenuated Anisakis simplex (L3) administration into Wistar rats: implication for anti-allergic reaction. Parasitol Res. 2015;114:2975-84.

17. Vericimo MA, Figueiredom I, Teixeira GA, Clemente SC. Experimental anisakid infections in mice. J Helminthol. 2015:89:620-4.

18. Chaligiannis I, Lalle M, Pozio E, Sotiraki S. Anisakidae infection in fish of the Aegean Sea. Vet Parasitol. 2012;184:362-6.

19. Anderson, RC. Nematode parasites of vertebrates. Their development and transmission. 2nd ed. CABI Publishing, Wallingford; 2000. 650 pp.

20. Perteguer MJ, Cuellar C. Isotype-specific immunoresponses in murine experimental anisakiasis. Zentrlbl Veterinarmed. 1998;B45:603-10.

21. Perteguer MJ, Rodero M, Flores JM, Dorea RC, Cuellar C. Cellular immune responses in mice immunized with Anisakis simplex larval antigens. Parasitol Res. 2001:87:396-404.

22. Kim JS, Kim KH, Cho S, Park HY, Cho SW, Kim YT, et al. Immunochemical and biological analysis of allergenicity with excretory secretory products of Anisakis simplex third stage larva. Int Arch Allergy Immunol. 2005;136:320-8.
23. Cho TH, Park HY, Cho S, Sohn J, Yoon YW, Cho JE, et al. The time course of biological and immunochemical allergy states induced by Anisakis simplex larvae in rats. Clin Exp Immunol. 2006;143:203-8.

24. Park SK, Cho MK, Park HK, Lee KH, Lee SJ, Choi HC, et al. Macrophage migration inhibitory factor homologs of Anisakis simplex suppress Th2 response in allergic airway inflamation model via CD4+CD25+Foxp3+Tcell recruitment. J Immunol. 2009;182:6907-14.

25. Nagano K. Gastric terranovasis. In: Ishikura H, Namki N, editors. Gastric anisakiasis in Japan. Tokyo: Springer-Verlag Academic; 1983. p. 133-6.

26. Desowitz RS. Human and experimental anisakiasis in the United States. Hokkaido lgaky Zasshi. 1986;61:358-71.

27. Kliks MM. Anisakiasis in the western United States: four new case reports from California. Am J Trop Med Hyg. 1983;32:526-32.

28. Mercado R, Torres P, Munoz V, Apt W. Human infection by Pseudoterranova decipiens (Nematoda, Anisakidae) in Chile: report of seven cases. Mem Inst Oswaldo Cruz. 2001;96:653-5.

29. Kobayashi Y, Kakemoto S, Shimakura K, Shiomi K. Molecular cloning and expression of a new major allergen Ani s 14 from Anisakis simplex. Food Hyg Saf Sci. 2015;56:194-9.

30. Anadón AM, Romarís F, Escalante M, Rodríguez E, Gárate T, Cuéllar C, et al The Anisakis simplex Ani s 7 major allergen as an indicator of true Anisakis infections. Clin Exp Immunol. 2009:156:471-8.

31. Dixon B, Walker B, Kimmins W, Pohajdakt B. Isolation and sequencing of a CDNA for an unusual hemoglobin from the parasitic nematode Pseudoterranova decipiens. Proc Nat Acad Sci USA. 1991;88:655-9.

32. González-Fernández J, Daschner A, Nieuwenhuizen NE, Lopata AL, Frutos CD, Valls A, et al. Haemoglobin, a new major allergen of Anisakis simplex. Int J Parasitol. 2015:45:399-407.

33. Nieuwenhuizen NE, Meter JM, Horsnel WG, Hoving JC, Fick L, Sharp MF, et al. A cross-reactive monoclonal antibody to nematode haemoglobin enhances protective immune responses to Nippostrongylus brasiliensis. PLoS Negl Trop Dis. 2013;29:2395-2400

34. Fernández-Caldas E, Quirce S, Marañón F, Diez Gómez ML, Gijón Botella H, López RR. Allergenic cross-reactivity between third stage larvae of Hysterothylacium aduncum and Anisakis simplex. J Allergy Clin Immunol. 1998;101:554-5.

35. Jones RE, Deardorff TL, Kayes SG. Anisakis simplex: histopathological changes in experimentally infected CBAIJ mice. Exp Parasitol. 1990;70:305-13.

36. Sastre J, Lluch-Bernal M, Quirce S, Arrieta I, Lahoz C, Del Amo A, et al. A double-blind, placebo-controlled oral challenge study with lyophilized larvae and antigen of the fish parasite Anisakis simplex. Allergy. 2000;55:560-4.

37. Alonso-Gómez A, Moreno-Ancillo A, López-Serrano MC, Suarez De-Parga JM, Daschner A, Caballero MT, et al. Anisakis simplex only provokes allergic symptoms when the worm parasitizes the gastrointestinal tract. Parasitol Res. 2004;93:378-84

38. Baeza ML, Rodríguez A, Matheu V, Rubio M, Tornero P, De Barrio M, et al. Characterization of allergens secreted by Anisakis simplex parasite: clinical relevance in comparison with somatic allergens. Clin Exp Allergy. 2004;34:296-302.

39. Audicana L, Audicana MT, De Corres LF, Kennedy MW. Cooking and freezing might not protect against allergenic reactions to ingested Anisakis simplex antigens in humans. Vet Record. 1997;140:235.

40. Kameyama R, Yagami A, Yamakita T, Nakagawa M, Nagase K, Ichikawa H, et al. A case of immediate allergy to Anisakis following saury intake. Japanese J Allergol. 2006:55:1429-32.

41. Vaickus LJ, Bouchard J, Kim J, Natarajan S, Remick DJ. Inbred and outbred mice have equivalent variability in a cockroach allergen-induced model of asthma. Comp Med. 2010;60:420-6. 